\title{
Análise epidemiológica da morbimortalidade por suicídio entre adolescentes em Minas Gerais, Brasil
}

\author{
Epidemiological analysis of morbidity and mortality from suicide \\ among adolescents in M inas Gerais, Brazil
}

\author{
M aria Leonor Ferreira A basse ${ }^{1}$ \\ Ronaldo Coimbra deOliveira ${ }^{2}$ \\ Tiago Campos Silva ${ }^{1}$ \\ Edinilsa Ramos deSouza ${ }^{3}$
}

\footnotetext{
${ }^{1}$ Coordenadoria de

Doenças eAgravos Não

Transmissíveis,

Superintendência de

Epidemiologia, Secretaria deEstado deSaúde de

$M$ inas Gerais. Av. Afonso

Pena 2300/90 andar,

Funcionários. 30130-007

Belo Horizonte M G.

cdnt@saude.mg.gov.br

${ }^{2}$ Coordenação-Geral de

Doenças eAgravos Não

Transmissíveis,

Departamento deAnálise

deSituação deSaúde,

Secretaria de Vigilância em

Saúde, M inistério da Saúde.

${ }^{3}$ Centro Latino-Americano

de Estudos de Violência e

SaúdeJ orge Careli,

Departamento de

Epidemiologia e M étodos

Quantitativosem Saúde,

Escola Nacional de Saúde

Pública Sérgio Arouca,

Fundação Oswaldo Cruz.
}

Abstract This study is a descriptive epidemiological analysis of morbidity and mortality from suicide in people aged 10 to 19 years living in the state of $M$ inas Gerais. The source of the mortality data were the data available in the M ortality Information System, DATASUS of the M inistry of $\mathrm{H}$ ealth, over the period 1980-2002. The morbidity data were collected from the Hospital Information System, over the period 1998-2003. Absolute and relative simple frequencies of 2.338 hospitalizations and 1.212 deaths from attempted suicide and suicide over the chosen period wereanalyzed according to sex, ageand method used. The population data used for calculating rates was extracted from the census conducted by the Brazilian Institute of $\mathrm{G}$ eography and Statistics. The International Classification of Diseases - ICD 9, from the years 1980 to 1995 and the 10th review since 1996, codes E950 to E959 and X60 to X84, were adopted respectively. The highest hospitalization rates were found among women. Self-poisoning was the most common method for attempting suicide in both sexes. However, adolescent males had the highest mortality rates and the most common methods of suicide (hanging and use of firearms) were more lethal than the method commonly chosen by females (self- poisoning). Based on these findings, the authors emphasize the need for preventing these events among young people.

Key words M ortality, Hospitalization, Suicide, Adolescents
Resumo Trata-se de estudo descritivo, com objetivo de realizar análise epidemiológica da morbimortalidade por suicídio na faixa etária de 10-19 anos de residentes em M inasGerais. Para os dados demortalidade, utilizou-se o Sistema de Informação sobre M ortalidade- DATASU S/M S, entre 1980 e2002, ede morbidade, o Sistema de Informação H ospitalar, entre 1998 e 2003. Analisaram-se freqüências simples absoluta e relativa de 2.338 internações e 1.212 óbitos, estratificadas por sexo, faixa etária e meios usados para perpetrar a auto-agressão. U tilizou-se para o cálculo das taxas a população extraída do censo e projeções intercensitárias do Instituto Brasileiro de Geografia eEstatística. Adotou-sea Classificação Internacional de Doenças 9a revisão ( 1980 e 1995) ea 10 a revisão a partir de 1996, códigos E950 a E959 e X60 a X84, respectivamente. Os resultados mostraram maior freqüência de internações entre mulheres. A auto-intoxicação foi o meio mais comum nas tentativas de suicídio para ambos os sexos. No entanto, foi observada maior mortalidade entre os homens, cujos princi pais meios escol hidos foram enforcamento earma defogo, formas maisletais do queas utilizadas pelas mulheres (intoxicação). Diante de tais achados, os autores destacam a necessidade de preven ção desseagravo junto à população de adolescentes.

Palavras-chave M ortalidade, H ospitalização, Suicídio, Adolescentes 
Introdução

O comportamento suicida pode ser entendido como "todo ato pelo qual um indivíduo causa lesão a si mesmo, qualquer que seja o grau de intenção letal e de conhecimento do verdadei ro motivo desse ato". Esta noção permite conceber o comportamento suicida como um continuum que inicia com pensamentos de autodestruição, passa das ameaças e gestos às tentativas de suicídio e, finalmente, consuma 0 ato suicida ${ }^{1}$.

Em grande parte do mundo, o suicídio é estigmatizado, rodeado de tabus, é marcado pelo "não dito". Dizer queuma pessoa morreu provoca comoção esolidariedade; entretanto, quando se diz quea morte foi provocada pelo suicídio, a fala fica suspensa, não circula, causando constrangimento. 0 assunto é evitado ou proibido, ficando uma mácu$1 a^{2}$. De modo geral, a população tende a negar essa atitude tão séria e grave contra o fluxo natural da vida, especialmente quando ocorre em adolescentes e, ainda mais, em crianças. A pesar disso, atualmentesabe-se queosadolescentes são especialmente vulneráveis a reagirem com atitudes suicidas em resposta a sérios conflitos que estejam passando ${ }^{3}$.

Segundo a Organização Mundial de Saúde (OM S) ${ }^{4} 0$ suicídio está entreas dez principais causas deóbito para todas as pessoas maiores de cinco anos de idade. Em todos os países onde há informações fidedignas sobre a mortalidade, esse evento encontra-se entre as três principais causas de morte para as pessoas de ambos os sexos com idade entre $15 \mathrm{e}$ 34 anos.

Diante disso, este trabalho tem como objetivo descrever o panorama epidemiológico da morbimortalidade por suicídio e tentativas, na faixa etária entre 10 e 19 anos, em M inas Gerais.

Aspectosfísicos, psíquicos esociais da adolescência: breves considerações

Pode-secaracterizar a adolescência como um período de mudanças profundas, uma fase de transição, de passagem da infância para a vida adulta, da sociabilidade da vida em família para as relações sociais mais amplas. As metamorfoses da puberdade provocarão alterações internas e externas, as transformações físicas repercutirão na vida psíquica e vice-versa. As mudanças corporais colocarão 0 adolescente às voltas com o corpo desconhecido, suspeito efonte deinquietude. Perde-se o corpo conhecido da primeira infância e em seu lugar surge um corpo que vive o impacto da maturação sexual. A sexualidade "antes calada, agora grita" e faz novas exigências, interpela e questiona o sujeito. Instala-se, então, um mal-estar ${ }^{5}$.

A adolescência também é vista como um momento de separação, de ruptura e de intenso trabal ho de luto, que estarão relacionados à renúncia da segurança da infância e de seu meio protetor, 0 distanciamento e a destituição da imagem heróica das figuras parentais. Este movimento poderá levar o jovem a buscar suas próprias respostas e a assumir a responsabilidade de seus atos, de seus interesses e de suas escolhas. Dessa forma, ele necessita mostrar para si mesmo e para os outros que pode ter idéias próprias, que pode pensar em sua vida e decidir os caminhos que irá tomar. Entretanto, esta posição não é fácil de ser assumida. Trata-se de uma fase delicada, pois o jovem oscila entre as novas reivindicações e manifestações de uma imaturidade própria da idade. Torna-se essencial que os pais apóiem seu filho nesta travessia, que não assumam a condução das escolhas deste, mas que lhe dêem coragem de ir adiante. Caberá ao pai ou ao seu substituto assumir o lugar da "lei ordenadora" da cultura, quando se fizer necessário ${ }^{5}$.

Em busca de respostas próprias, a ligação a um "melhor amigo", a um grupo ou a uma turma pode ser outra maneira de lidar com estas vivências e fazer frente ao "mal-estar" instalado. Junto de seus pares, busca-se o jeito adolescente de ser, com gosto e estilo próprio; identificações; novas maneiras de lidar com os paradoxos vividos e a elaboração de normas, regras e valores. N este momento, o jovem precisa ir além dos ideais preconizados pela cultura. Faz-se necessária a transgressão dos mesmos, mas atualmente, nota-se a banalização de tais ideais. N este movimento, muitas vezes, o corpo torna-se o objeto da transgressão, através de tatuagens, piercing em várias partes corporais, tinturas nos cabelos em tons inusitados. 0 corpo não fala, age, podendo aparecer vários sintomas, como anorexia, bulimia, toxicomania. A gravidez precoce pode se apresentar como uma saída para o impasse do "mal-estar" em relação ao sexual. 0 isolamento, o ensimesmamento podem ser mecanismos de defesa frente às solicitações do processo de adolescer. N esse contexto, caso o par parental se licencie de suas funções simbólicas e 0 adolescentefique completamentesó nestemomento de transição, ele pode substituí-los por um sintoma, cujo efei to catastrófico não surge de imediato, até que se produza uma lesão, uma mutilação ou mesmo a morte ${ }^{5,6}$.

O jovem, na busca por seus pares, pode estabelecer ligações que criem laços sociais, mas também pode propiciar encontros com um tirano que 
o conduza à destrutividade. Por meio dele, pode-se chegar à delinqüência, à drogadição, à violência e tantas outras formas de marginalização, que assinalam a precariedade dos laços e dos processos simbólicos 5 .

Em síntese, na adolescência, existem vários componentes físicos e psicossociais neste processo de transformação. Dentre eles, destacam-se a busca de si mesmo e da identidade; a separação progressiva dos pais; a vinculação ao grupo; a evolução da sexualidade; atitudesocial reivindicatória, variações freqüentes do humor e contradições sucessivas. Além destes, há os comportamentos de risco que se originam da necessidade de experimentar o novo e desafiar o perigo. Impulsionado pelo mito da indestrutibilidade, o jovem pode acreditar que "nada demal Iheacontecerá eque dará conta de controlar tudo". Esta tendência a agir impulsivamente como forma de solucionar a crise instaurada poderá levar esse jovem a fazer escolhas sem considerar a possibilidade de causar danos, no presente ou no futuro, a si mesmo e/ou a outrem.

\section{Epidemiologia do suicídio}

A OM S7 considera que o suicídio éum importante problema de saúde pública. Os dados mundiais relativos ao ano de 2000 mostram que aproximadamente um milhão de pessoas cometeram suicídio; a cada 40 segundos, uma pessoa morre por essa causa; a cada 3 segundos, uma pessoa atenta contra a própria vida; para cada suicídio, existem pelo menos dez tentativas, suficientemente sérias a ponto de exigir atenção médica; para cada tentativa registrada, ocorrem outras quatro desconhecidas; cada suicídio tem um sério impacto em pelo menos outras seis pessoas; o impacto psicológico, social efinanceiro em uma família e comunidadeé imensurável.

Segundo o Informe Mundial sobre a Violência e Saúde, os índices mais altos de suicídio se registram em países da Europa O riental - destacandose, entre eles, Hungria, Finlândia e Japão - e os mais baixos na América Latina, países muçulmanos e asiáticos ${ }^{8}$.

No Brasil, no ano de 2000, segundo Gawryszewski et al. ${ }^{9}$, o suicídio foi responsável por 6.778 óbitos, que corresponderam a 5,7\% do total de mortes, com taxa de mortalidade igual a 4,0/ 100 mil habitantes. Sendo que, entre os homens, a taxa de mortalidade foi de 5,0 e, para as mulheres, de 1,6/100 mil habitantes.

A American Association of Suicidology ${ }^{10}$ estima que ocorram cerca de cem a duzentas tentati- vas para cada suicídio entre jovens. A porcentagem desuicídio em jovens tem aumentado em todo o mundo. A taxa de suicídio nos Estados Unidos, no ano de 2000, foi de 12,0/100 mil habitantes, para jovens na faixa etária de 15 a 24 anos, sendo considerada a terceira causa de morte nessa população depois dos acidentes e homicídios ${ }^{11}$.

Souza et al. ${ }^{12}$ observaram no Brasil um crescimento de 35,3\% na taxa de mortalidade por suicídio entre jovens de 15 a 24 anos em suas principais capitais. A cidade de Belo Horizonte apresentou taxa de mortalidade de 4,3 no ano de 1979 e 6,8/ 100.000 habitantes em 1995; portanto, um crescimento de $58,1 \%$ no período estudado. D 'O liveira ${ }^{13}$ apresentou dados preliminares deum levantamento realizado pelo Núcleo deAtenção ao Suicídio da Secretaria Municipal de Saúde do Rio de Janeiro, no período de agosto de 2000 a agosto de 2003 , em onze unidades de saúde e dois hospitais gerais deste município. Observou-se que $60 \%$ das notificações foram de indivíduos com até 34 anos, sendo a maior incidência na faixa de 15 a 24 anos (30,4\%) e igualmente distribuída entre os sexos.

A Análise da Situação de Saúde em M inas Gerais, realizada pela Superinten dência deEpidemiologia/SES-M G, mostrou um aumento nas taxas de mortalidade por suicídio nos jovens de 15 a 29 anos, de $47,5 \%$, entre 2000 e 2004 , sendo que, neste último ano, passou a ser o segundo grupo etário de maior risco de morte por essa causa ${ }^{14}$.

Fazem-se necessários alguns cuidados ao se analisar dados sobre suicídio, pois existem algumas questões que interferem no real dimensionamento deste problema. Entre elas, podemos citar: tendência à subnotificação de dados; falhas nos registros; taxas oficiais precárias; a imprecisão na fonte de produção (Polícia, Institutos de M edicina Legal - IML). Além disso, há um estigma que cerca este tipo de morte, o uso de conceitos e definições diversas do ato suicida, a dificuldade em saber se o episódio foi acidental ou intencional. Em se tratando de crianças e adolescentes, essas barreiras na notificação serão mais acentuadas, na medida em que os atos autodestrutivos serão negados ou até escondidos pela família, devido a sentimentos de culpa e/ou vergonha ${ }^{15,16}$.

Fatores de risco e de proteção para o suicídio

Entre os adolescentes, demodo geral, os principais fatores de risco para o suicídio são: idade, tentativa prévia, transtorno de humor, depressão, abuso de drogas lícitas eilícitas, ausência deapoio familiar, história familiar de doenças psiquiátricas, his- 
tória familiar de comportamento suicida, doença física gravee/ou crônica, eventos estressores, orientação sexual|17-21.

A baixa auto-estima, os conflitos familiares, 0 fracasso escolar, as perdas afetivas são sintomas que, associados às condições de estresse emocional, podem colocar os jovens em grupo de risco para o suicídi ${ }^{22}$. A autora ainda assinala que mais importante do que procurar a causa do problema é identificar como seus efeitos são vividos no contexto sócio-familiar, qual a função do significado que o sintoma adquire no contexto das interações onde ele se produz e se mantém.

Em relação à idade, é importante ressaltar que o suicídio entre os jovens vem crescendo em números alarmantes. Os estudos realizados por Cassorla et al. ${ }^{23}$ revelaram que estamos assistindo a um aumento concomitante da violência por homicídio e por suicídio na faixa etária entre 15 e 39 anos. Q uanto ao sexo, vemos que os homens apre sentam uma freqüência de suicídio três vezes maior do que as mulheres. Essa relação é constante nas diferentes faixas etárias; contudo, para o sexo feminino, a chance de cometer tentativas de suicídio é de três a quatro vezes maior ${ }^{1}$.

De acordo com Lippi ${ }^{24}$, a violência psicológica e/ou sexual sofrida na infância/ adolescência constitui outro importante fator de risco, assim como a depressão, a desesperança e a ideação suicida.

É importante salientar que são freqüentes os indícios sobre a intenção suicida.

Segundo Silva ${ }^{25}$, o suicídio deve ser visto mais como um ato de comunicação do que como um gesto único. 0 autor afirma que esse ato representa uma comunicação para uma sociedade que 0 impediu de comunicar-se de outras formas. Muitos adolescentes se expressam direta ou indiretamente com bilhetes, falas, isolamento e intolerância à dor. Entretanto, esses indícios nem sempre são percebidos, sendo, às vezes, negados por familiares, amigos ou pessoas próximas ${ }^{26}$.

À medida queavança o conhecimento sobreos fatores de risco, também se tornam cada vez mais necessárias a pesquisa, a identificação e a confirmação dos fatores protetores para este comportamento, tanto no nível individual como coletivo. Diversos países, como Finlândia, N oruega, Suécia, Austrália, N ova Zelândia, França e Brasil têm como preocupação o desenvolvimento de estratégias de prevenção do suicídio em diferentes populações ${ }^{27}$. Os principais fatores que desempenham essa função são: boa relação e apoio familiar; bons relacionamentos sociais; senso de propósito para a própria vida; capacidade para receber conselho e bus- car ajuda quando surgem problemas; adoção de val ores etradições culturais; integração social. Torna-se importante o reconhecimento desses fatores pelos profissionais de saúde, viabilizando o acesso às várias intervenções clínicas e apoio para a busca deajuda, principalmente, aos grupos de risco mais vulneráveis, como portadores de transtornosmentais e/ou usuários de drogas lícitas e lícitas ${ }^{1}$.

Ao considerar os fatores de risco e de proteção para o suicídio, não se pode perder de vista que toda tentativa de suicídio, em especial de um adolescente, é dirigida a al guém e expressa uma necessidade de afeto, de amor, de ser ouvido e reconhecido como pessoa. Deveser interpretada como uma pergunta que requer resposta ${ }^{28}$.

\section{M etodologia}

Este estudo realizou uma análise epidemiológica descritiva dos dados referentes à mortalidade por suicídio e às internações hospitalares por tentativas de suicídio de residentes em M inas Gerais, na faixa etária de 10 a 19 anos, de ambos os sexos. Para estudar a mortalidade, a fonte de dados adotada foi o Sistema de Informação sobre M ortalidade (SIM), M inistério da Saúde/D epartamento deAnálise eTabulação de Dados do Sistema Ú nico de Saúde (DATASUS) no período de 1980 a 2002. Para as internações, utilizou-se o Sistema de Informações Hospitalares-SIH/SUS/DATASUS, no período de 1998 a 2003. Considera-se que os dados desse sistema representem $80 \%$ das internações realizadas no país ${ }^{29}$.

Os dados foram analisados em termos de freqüências simples absoluta (números absolutos) e relativa ( proporções, razão e taxas). Para o cálculo das taxas de mortalidade e de internação, foram considerados como numerador o número demortes e internações e como denominador a população dos censos de 1980, 1991 e 2000 e a contagem populacional de 1996. Para os demais anos, foram utilizadas as estimativas populacionais intercensitárias de 10 de julho (IBGE) do referido ano.

Adotou-se a ga revisão da Classificação Internacional deDoenças para o período de 1980 a 1995 ea $10^{a}$ revisão a partir do ano de 1996. Em ambas, o suicídio e as tentativas de suicídio encontram-se no agrupamento de Causas Externas, capítulo XX. $\mathrm{Na}$ primeira, com a denominação de "lesões autoinflingidas" sob os códigos E950-E959 e, na segun$\mathrm{da}$, como "lesões autoprovocadas voluntariamente", com os códigos X60 a X84. 


\section{Resultados ediscussão}

Em M inas Gerais, entre 1998 e 2003, o número total de internações no SU S decorrentes detentativas de suicídio foi de 14.443 , das quais $55,4 \%$ eram do sexo masculino e $44,6 \%$, do feminino. A faixa etária dos 10 aos 19 anos representou $16,2 \%$ do total dessas internações. De acordo com os dados encontrados nesse estudo, notou-se que as taxas de internação no sexo feminino foram superiores (5,8 e 19,9/ 100.000 habitantes entre 10 e 14 anose 15 a 19 anos, respectivamente) a dos homens (4,0 e 12,4/ 100.000 habitantes (Figura 1).

Esses achados estão de acordo com o encontrado pelo Departamento de Saúde do Estado de Indiana, nos Estados Unidos ${ }^{30}$, em seu relatório de 2005, sobre lesões fatais e não fatais, cujas taxas foram de 3,4 e 16,5 internações por 100 mil habitantes em homens emulheres com idadeentre $10 \mathrm{e}$ 14 anos, respectivamente. $N$ a faixa etária de 15 a 19 anos, os resultados foram: 37,3 internações para homens por 100 mil habitantes e, para mulheres, 77,2. Em M assachusetts, o Departamento de Saúde Pública ${ }^{31}$ divulgou que, no período de 1996 a

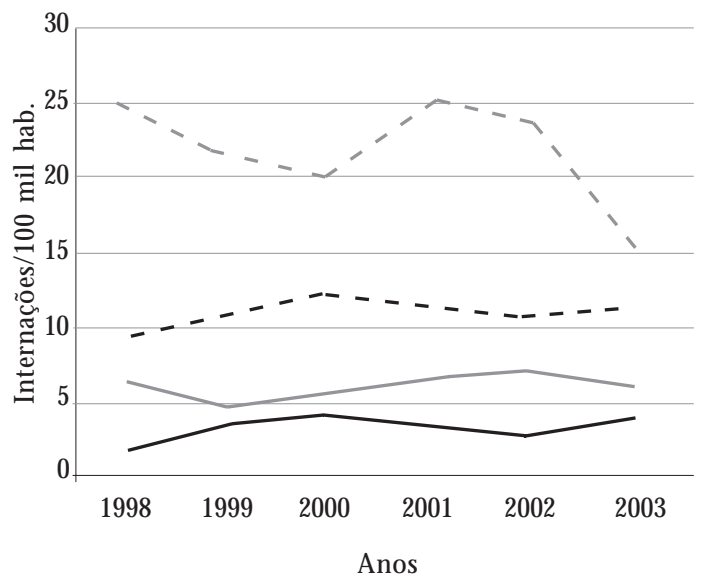

$$
\begin{aligned}
& 10 \text { a } 14 \text { anos Masc } \\
& 10 \text { a } 14 \text { anos Fem } \\
& \text { - } 15 \text { a } 19 \text { anos M asc } \\
& - \text { - } 15 \text { a } 19 \text { anos Fem }
\end{aligned}
$$

Figura 1. Taxa de internação por lesões autoprovocadas, segundo sexo e faixa etária. Minas Gerais, 1998-2003.
1998, as taxas médias anuais também foram mais elevadas em mulheres com idades entre 15 e 19 anos (148,3/100 mil habitantes) em relação aos demais grupos citados acima. Segundo Marquet et al. ${ }^{32}$, num serviço sentinela holandês, as mulheres entre 10 e 19 anos tentaram mais suicídios de 1983 a 2003. Entretanto, em algumas localidades como Oakland, Califórnia foi observada uma inversão do risco de internação devido a tentativas de suicídio por sexo, uma vez que os homens com 15 a 24 anos apresentaram taxas duas vezes maiores que as das mulheres nos anos de 1998 a 2000 ${ }^{33}$.

No Brasil, no ano de 2000, foi observada uma taxa maior de internação por tentativa de suicídio nos homens (6,8/ 100.000 habitantes) do que nas mulheres (4,2/ 100.000 habitantes), da população geral. Ao se estratificar os dados por sexo e faixa etária, o sexo feminino apresentou maiores taxas de internação do que o masculino, nos grupos de 10 a 14 anose 15 a 19anos (2,7 e 6,8/ 100.000 habitantes, respectivamente). Entre os homens, as taxas alcançaram os seguintes valores: 10 e 14 anos (2,5/100.000 habitantes) e 15 a 19 anos (5,5/ 100.000 habitantes) ${ }^{16}$.

A Figura 2 mostra que, em relação ao método, a auto-intoxicação foi o mais utilizado para a tentativa de cometer suicídio em ambos os sexos, principalmente pelas mulheres que optaram três vezes mais por esse meio que os homens. Estes achados estão em consonância com os resultados obtidos por Avanci ${ }^{34} \mathrm{em}$ um estudo realizado em Ribeirão Preto. A autora observa o predomínio de $77,8 \%$. do sexo feminino nos atendimentos hospitalares. Os métodos mais utilizados para ambos os sexos foram a ingestão de medicamentos $(65,3 \%)$ e de substâncias químicas (20,8\%).

Em M inas Gerais, no Sistema Ú nico de Saúde (SUS), as lesões decorrentes de objetos cortante ou penetrante foram a segunda causa de internações por tentativas de suicídio (Figura 2). Em O regon, Andrus et al. ${ }^{35}$ encontraram que $75,5 \%$ dos adolescentes de 10 a 17 anos escolheram como método o uso abusivo de drogas e $10,4 \%$ a laceração ou perfuração. Bahls ${ }^{36}$ encontrou que aproximadamente $80 \%$ dos adolescentes que tentaram suicídio empregaram a ingestão de substâncias que provocaram intoxicações exógenas, seguida decorte nos pulsos.

Diversos autores, entreeles, Cassorla ${ }^{37} ; \mathrm{K}$ achur et al. ${ }^{38}$ e Larraguibel et al. ${ }^{39}$ relatam haver uma associação do sexo com o método utilizado para tentar ou cometer o suicídio. Os homens optam por métodosmaisletais (enforcamento etiro por arma de fogo) do que as mulheres. 
Em relação a mortalidade, ocorreram, em M inas Gerais, 25.060 óbitos por causas externas de adolescentes, entre 10 e 19 anos, no período de 1980 a 2002 (uma mulher para cada três homens), sendo $4,8 \%$ destes decorrentes de suicídio. Deacordo com a Figura 3, o sexo masculino, na faixa de 15 a 19 anos, apresentou riscos mais elevados de morte ao longo dos anos, com uma taxa de mor-

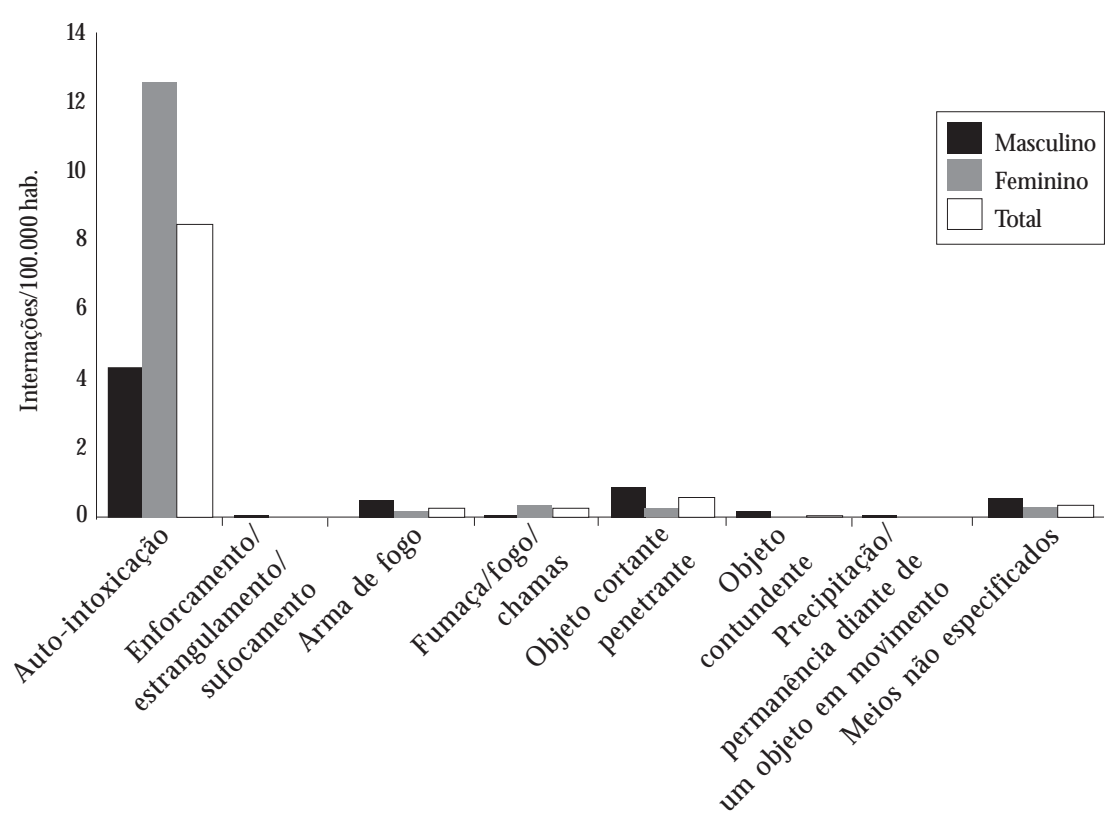

Figura 2. Taxa de internação por lesões autoprovocadas, segundo meio utilizado e sexo. M inas Gerais, 1998-2003.

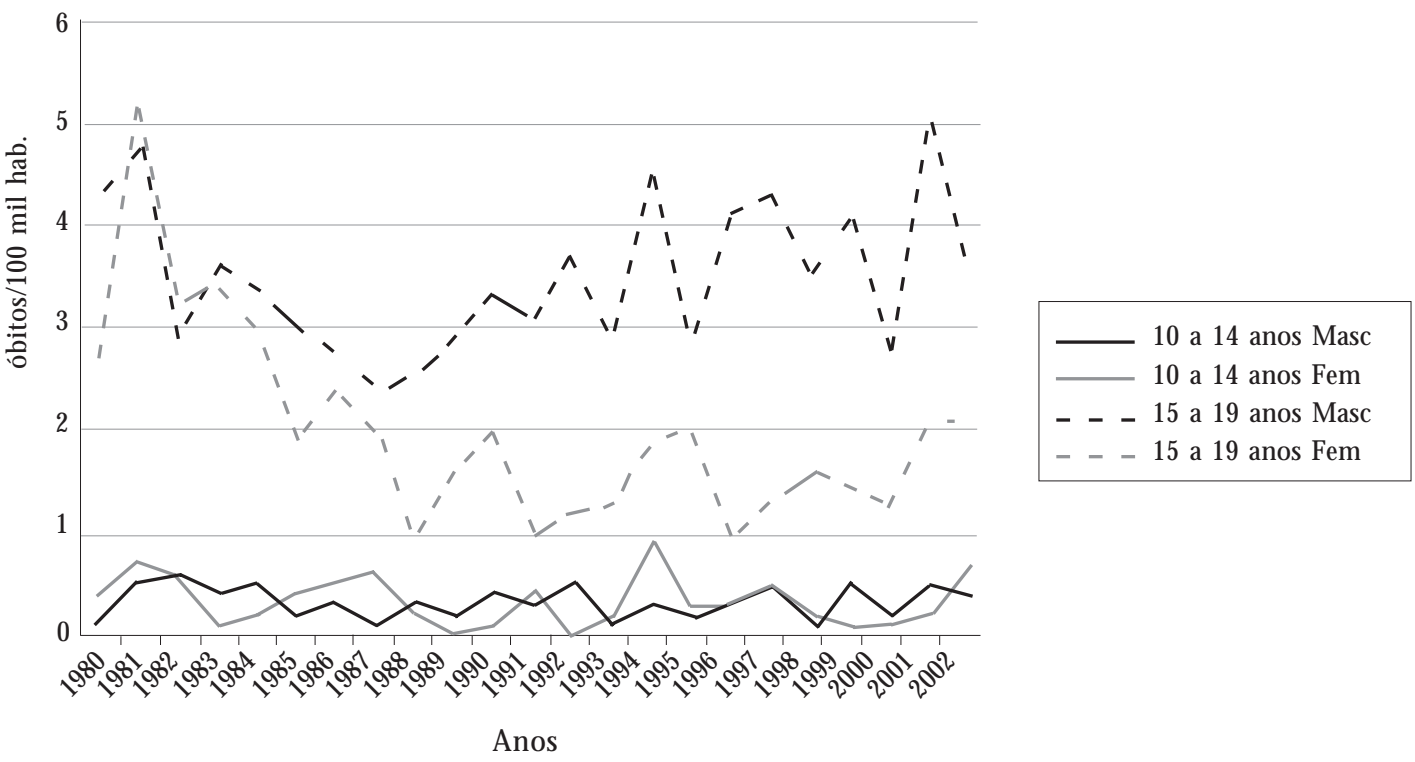

Figura 3. Taxa de mortalidade por lesões autoprovocadas, segundo faixa etária e sexo. M inas Gerais, 1990-2002. 
talidade média aproximadamente duas vezes maior que o feminino (3,5 e 2,0/ 100.000 habitantes). No entanto, as taxas de mortalidade para os jovens de 10 a 14 anos apresentaram mais oscilações entre os sexos. Esses achados estão em conformidade com o estudo realizado na Argentina por Basil ${ }^{40}$ entre jovens de 15 a 24 anos. Os resultados demonstraram que os homens apresentaram maior risco de morte por suicídio do que as mulheres (19,2 e 5,5/ 100.000 habitantes, respectivamente), tanto na República Argentina quanto nas províncias. Na Austrália, no período de 1990 a 1994, foram encontradas taxas de mortalidade por suicídio de 25,7 no sexo masculino e 5,1/ 100.000 habitantes no feminino, em jovens de 15 a 24 anos $^{41}$.

$\mathrm{NaFigura} 4$, pode-seobservar que entre os anos de 1996 e 2002, o principal meio utilizado para a conclusão do ato suicida por homens foi o enforcamento/estrangulamento/sufocamento (1,1 óbito/ 100.000 habitantes), seguido por arma de fogo (0,6 óbito/ 100.000 habitantes), seguido por arma defogo (0,6óbito/ 100.000 habitantes). Entreas muIheres, os meios foram enforcamento/ estrangulamento/ sufocamento e auto-intoxicação, com taxas iguais de 0,3 óbito/100.000 habitantes.
Ao se comparar vários estudos sobre tentativas e suicídios entre adolescentes, notou-se variações entre as taxas de internações e de mortalidade, quanto a sexo e faixa etária, nos vários países. Qualquer taxa, por menor que seja, não deve ser negligenciada, pois a população estudada trata-se de jovens com intenso sofrimento que, já iniciando a vida, estariam desistindo del $a^{42}$.

\section{Conclusão}

0 presente estudo aponta que, pelos critérios internacionais, M inas Gerais apresentou baixa taxa de mortalidade por lesões autoprovocadas voluntariamente em adolescentes (taxas inferiores a 5 óbitos/ 100.000 habitantes). 0 Estado reproduziu, tanto para as tentativas de suicídio, quanto para o ato consumado, a realidade de diversos países. As taxas de internação foram mais altas entre as jovens, numa razão de três para cada homem jovem, sendo a auto-intoxicação o meio mais utilizado para a tentativa de cometer suicídio em ambos os sexos, principalmente pelas mulheres. Estes dados sugerem afacilidade deacesso ao meio, prin-

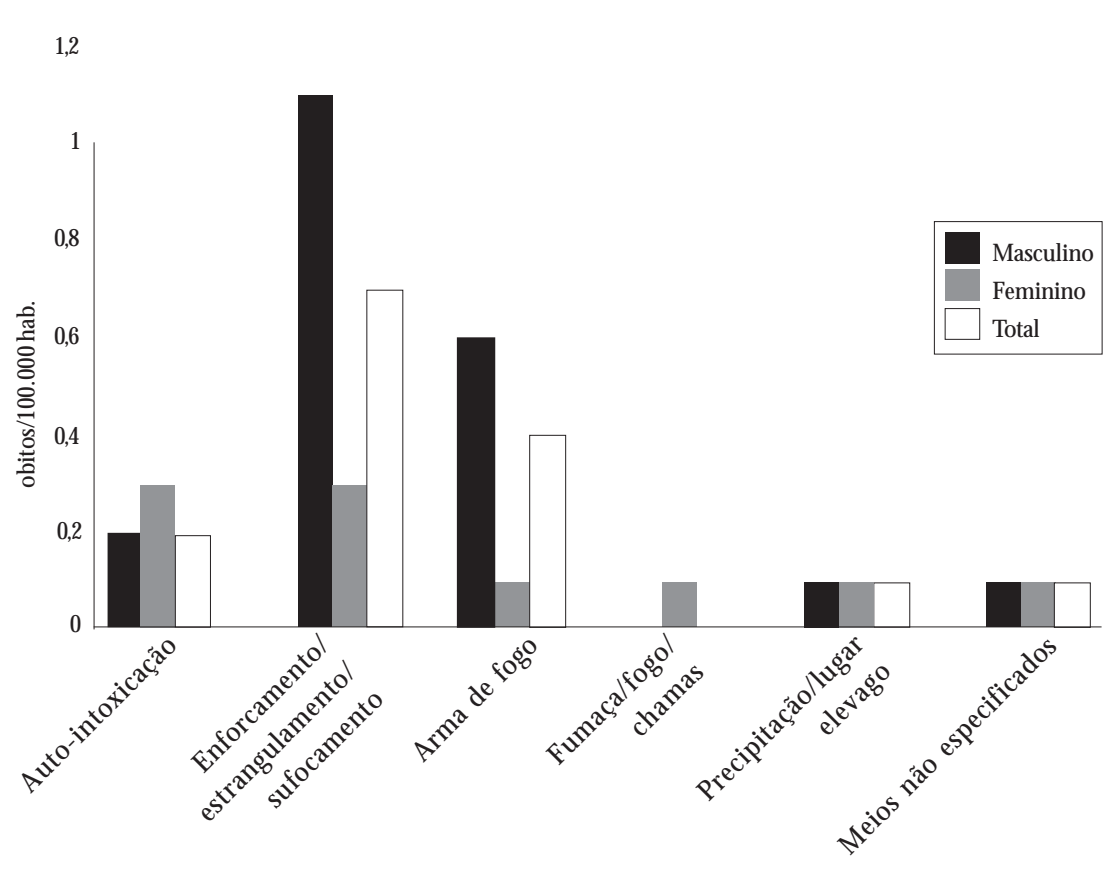

Figura 4. Taxa de mortalidade por lesões autoprovocadas, segundo meio utilizado e sexo. M inas Gerais, 1996-2002. 
cipalmente no caso das medicações. Isso nos remete a uma característica importante da sociedade atual, que é a busca da solução imediata para os conflitos emocionais. Acredita-se que haja remé dio para tudo. Assim, observam-se a banalização eo uso indiscriminado demedicamentos, entreeles, os antidepressivos e ansiolíticos.

Os nossos resultados mostram que os adolescentes masculinos apresentaram riscos mais elevados de morte ao longo dos anos, com uma taxa de mortalidade média aproximadamente duas ve zes maior que o sexo feminino. 0 principal meio utilizado para a conclusão do ato suicida, tanto por homens quanto por mulheres, foi o enforcamento, seguido pelo estrangulamento ou sufocamento, lesões por arma defogo em homens eautointoxicação em mulheres.

Esses resultados nos mostram que é necessário aprofundar os estudos sobre o problema, de forma a ampliar o conhecimento acerca desse tema, as manifestações suicidas na adolescência.

A morte dos jovens por causas violentas, entre elas, o suicídio, éum dos problemas atuais no mundo e também no Brasil. 0 suicídio éum fenômeno que sofre a interferência de fatores individuais, ambientais, sociais. Seja na forma de tentativa ou do ato consumado, expressa uma dor emocional que o sujeito considera ser interminável, intolerável e com a qual acredita não ter capacidade de lidar. Esse fenômeno desafia a todos: pais, educadores, profissionais de saúde, como também o campo das ciências humanas e sociais. Não bastam médicos, enfermeiras, auxiliares de enfermagem epsicólogos melhorarem a qualidade de seusatendimentos. Fazse necessário que profissionais da saúde e da educação empreendam um trabalho multidisciplinar. Torna-se essencial ir além de aspectos curativos, é imprescindível que se consiga atingir a dimensão da prevenção. Pois, só assim, será possível estruturar uma atenção que abranja não só os adolescentes que tentaram suicídio e/ou apresentaram comportamentos de risco. Buscar estratégias que possam minimizar as situações ou vivências estressoras, em especial as que se relacionam à família ea escola, é fundamental, pois esses espaços podem funcionar como fatores de risco, mas também podem atuar como fatores de proteção.

A sensibilização e a capacitação de profissionais para assistir a este grupo etário e a organização de uma rede de assistência efetiva são primordiais para 0 alcance de tal objetivo.

\section{Colaboradores}

M LF A basse, RC deOliveira, T Campos-Silva eER de Souza participaram igualmente de todas as etapas da elaboração do artigo. 
1. Werlang BG, Botega NJ. Comportamento suicida. Porto Alegre: Artmed; 2004

2. Galias I. Aspectos psicodinâmicos do suicídio. Simpósio Internacional de Suicídio: Avanços e Atualizações. São Paulo; 2004.

3. American Academy of Pediatrics. Suicide and suicide attempts in adolescents. Pediatrics 2000; 105(4):871-874.

4. Organização Mundial da Saúde. Prevenção do suicídio: manual para médicos clínicos gerais. Genebra: OM S; 2000.

5. Curi TCGB. A adolescência em questão. Rev Griphos 1998; 16:78-82.

6. Dias S. A inquietante estranheza do corpo e o diagnóstico na adolescência. Psicol USP 2000; 11(1):119135.

7. Organización Mundial de la Salud. Prevención del suicidio: un instrumento para docentes y demás personal institucional. Ginebra: OM S; 2001.

8. Organización Mundial de la Salud. Informe mundial sobre la violencia y la salud (sinopsis). Ginebra: OM S; 2002.

9. Gawryszewski VP, Koizumi MS, Mello-Jorge MHP. As causas externas no Brasil no ano 2000: comparando a mortalidade e a morbidade. Cad Saúde Pública 2004; 20(4):995-1003.

10. American Association of Suicidology. USA Suicide: 2004 Official Final Data. Washington; 2006. [cited 2007 Ago 15]. Available from: http://www.suicidology.org/ associations/1045/files/2004datapgv1.pdf

11. Bedolla RIV, Musacchio AB, Ramirez RP. Suicidio en Jóvenes. Rev Fac M ed UNAM 2005; 48(2):54-57.

12. Souza ER, M inayo MCS, Malaquias JV. Suicide among young people in selected Brazilian State Capitals. Cad Saúde Pública 2002; 18(3):673-683.

13. D`Oliveira CF. Atenção a jovens que tentam suicídio: é possível prevenir. In: Ministério da Saúde. Violência faz mal à saúde. Brasília: Ministério da Saúde; 2004. p. 177-184.

14. Abasse MLF, Campos-Silva T, Machado EL, Botelho JMA, Belo JA, Lima OMG, Costa HF, Bicalho TM. $M$ orbimortalidade por doenças e agravos não transmissíveis. In: Superintendência de Epidemiologia. Análise da situação de saúde M inas Gerais. Belo Horizonte: Secretaria de Estado de Saúde de M inas Gerais; 2007. p. 121-158.

15. Cassorla RM S, Smeke ELM . Autodestruição humana. Cad Saúde Pública 1994; 10(1):61-73.

16. M inayo M CS. Suicídio: violência auto-infligida. In: Secretaria de Vigilância em Saúde. Impacto da violência na saúde dos brasileiros. Brasília: M inistério da Saúde; 2005. p. 205-239.

17. Bahls SC. Suicídio em crianças e adolescentes. In: Bahls SC. A depressão em crianças e adolescentes e 0 seu tratamento. São Paulo: Lemos Editorial; 2004. p. 71-78.

18. Souza ER. Avanços do conhecimento sobre causas externas no Brasil e no mundo: enfoque quanti e qualitativo. In: Minayo MCS, Souza ER, organizadores. Violência sob o olhar da Saúde: infrapolítica da contemporaneidade brasileira. Rio de Janeiro: Fiocruz; 2003. p. 131-160.

19. Grossman DC, Milligan BC, Deyo RA. Risk factors for suicide attempts among Navajo adolescents. Am J Public Health 1991; 81(7):870-874.
20. Manson SM, Beals J, Dick RW, Duclos C. Risk factors for suicide among Indian adolescents at a boarding school. Public Health Rep 1989; 104(6): 609-614.

21. Pinhey TK, Millman SR. Asian/Pacific islander adolescent sexual orientation and suicide risk in Guam. Am J Public Health 2004; 94(7):1204-1206.

22. Teixeira CM FS. Tentativas de suicídio na adolescência. Rev UFG [periódico na Internet]. 2004 Jun [acessado 2006 mai 15]; 6(1):[cerca de 6.]. Disponível em: http://www.proec.ufg.br/revista_ufg/juventude/ suicidio.html

23. Cassorla RM S, Smeke ELM . Autodestruição humana. Cad Saúde Pública 1994; 10 (Supl 1):61-73.

24. Lippi JRS. Tentativas de suicídio associada à violência física, psicológica e sexual contra a criança e 0 adolescente [tese]. Rio de Janeiro (RJ): Fiocruz; 2003.

25. Silva M M. Suicídio: trama da comunicação [dissertação]. São Paulo (SP): Pontifícia Universidade Católica; 1992.

26. M eleiro AM . O paciente suicida no Hospital Geral. In: Renério FJ , M eleiro AM A, M archetti RL, Henriques JS. Psiquiatria e psicologia no hospital geral: integrando especialidades. São Paulo: Lemos, 1997. p. 245-283.

27. Botega NJ, Werlang BSG, M acedo M M K. Prevenção do comportamento suicida. Psico 2006; 37(3): 213-220.

28. Serfaty E. Suicidio em la adolescencia. Adolesc Latinoam 1998; 1(2):105-110.

29. Lebrão ML, Mello-Jorge MHP, Laurenti R. Morbidade hospitalar por lesões e envenenamentos. Rev. Saúde Pública 1997; 31(Supl 4):26-37.

30. Beck S, Graves $C$. Injuries in Indiana: A Report on Injury-Related Fatalities and Injuries Resulting in Hospitalization. Indianapolis: Indiana State Department of Health; 2005.

31. Bureau of Family and Community Health. Suicide and self-inflicted injury in M assachusets: 1996-1998. Boston: M assachusetts Department of Public H ealth; 2001.

32. M arquet RL, Bartelds AIM, Kerkhof AJFM, Schellevis FG, van der Zee J. The epidemiology of suicide and attempted suicide in Dutch general practice 19832003. BM C Family Practice [serial on the Internet] $2005 \mathrm{~N} \mathrm{ov}$ [cited 2007 Ago 10]; 6:45 [about 7 p.]. Available from: http://www.biomedcentral.com/content/ pdf/1471-2296-6-45.pdf

33. M urgai N. Oakland Health Profile 2004. Oakland: Alameda County Public Health Department; 2004.

34. Avanci RC. 0 adolescente que tenta suicídio: estudo epidemiológico em uma unidade de urgência [dissertação]. Ribeirão Preto (SP): Universidade de São Paulo; 2004.

35. Andrus JK, Fleming DW, Heumann MA, Wassell JT, Hopkins DD, Gordon J. Surveillance of Attempted Suicide among Adolescents in Oregon, 1988. AM J Public Health 1991; 81:1067-1069.

36. Bahls SC. Aspectos clínicos da depressão em crianças e adolescentes. J Pediatr 2002; 78(5):359-366.

37. Cassorla RMS. Comportamentos suicidas na infância e adolescência. J Bras Psiquiatr 1987; 36:137-144.

38. Kachur SP, Potter LB, Powell KE, Rosenberg ML. Suicide: Epidemiology, prevention and treatment. Adolesc M ed 1995; 6(2):171-182.

39. Larraguibel M, González P, Martínez V, Valenzuela $R$. Factores de riego de la conducta suicida en niños y adolescentes. Rev Chil Pediatr 2000; 71(3):1-13. 
40. Basile HS. El suicidio de los adolescentes en Argentina. Alcmeon Rev Arg Clín Neuropsiquiátr 2005; 12(3):211-231.

41. Cantor $\mathrm{CH}$, Neulinger K, Leo DD. Australian Suicide Trends 1964-1997: youth and beyond? MJA 1999; 171:137-141.

42. Barros MDA, Ximenes R, Lima M LC. Mortalidade por causas externas em crianças e adolescentes: tendências de 1979 a 1995. Rev. Saúde Pública 2001; 35(2):142-149.

Artigo apresentado em 26/04/2006

Aprovado em 04/12/2007

Versão final apresentada em 20/03/2008 